\title{
Post-Discharge Follow-Up of Preterm Infants at High-Risk Neonatal Follow-Up Clinic of a Maternity Hospital
}

\author{
Naeeme Taslimi Taleghani ${ }^{1}$, Minoo Fallahi ${ }^{1{ }^{*}}$, Zahra Soltanttooyeh ${ }^{1}$, Ahmad Reza Shamshiri ${ }^{2,3}$ and \\ Mitra Radfar ${ }^{4}$ \\ ${ }^{1}$ Neonatal Health Research Center, Research Institute for Children's Health, Shahid Beheshti University of Medical Science, Tehran, Iran \\ ${ }^{2}$ Department of Community Oral Health, School of Dentistry, Tehran University of Medical Sciences, Tehran, Iran \\ ${ }^{3}$ Research Center of Tooth Caries Prevention, Research Center of Dentistry Sciences, Tehran University of Medical Sciences, Tehran, Iran \\ ${ }^{4}$ Imam Hossein Hospital, Shahid Beheshti University of Medical Science, Tehran, Iran \\ "Corresponding author: Neonatal Health Research Center, Research Institute for Children's Health, Shahid Beheshti University of Medical Science, Tehran, Iran. Email: \\ minoofallahi@yahoo.com
}

Received 2019 May 12; Revised 2019 June 24; Accepted 2019 September 22.

\begin{abstract}
Background: Recent advances in medical sciences have increased the survival of premature infants. Long-term follow-up is very important for decreasing the consequences of prematurity.

Objectives: The present study aimed at investigating common post-discharge problems of premature neonates.

Methods: In this prospective descriptive study, we selected preterm infants with a gestational age of $<34$ weeks or birth weight of $<$ 2,000 g referring to a high-risk neonatal follow-up clinic of Mahdieh Hospital from 2016 to 2017. Growth indices and other medical problems were evaluated.

Results: Of 140 newborns, 51.4\% were males. The mean gestational age and birth weight were 30 weeks and 1,366.99 g, respectively. The mean interval between the discharge and the referral time to the clinic was 6.16 days with a range of 1 to 45 days. The maximum number of visits of a patient to the clinic was 18 times. The first visit occurred at $2-3$ days after the discharge and the oldest age of patients at outpatient visits was 36 months. Although the growth level of most patients at birth was in the 50th percentile of growth charts, the impairment of growth indices was detected after discharge, particularly in neonates with birth weights of $<$ $1,000 \mathrm{~g}$. The rate of exclusively breastfed infants was $17 \%$ while $83 \%$ were fed by the formula. Re-admission was observed in $26.4 \%$. Infantile colic, gastroesophageal reflux, respiratory allergy, and hypothyroidism were observed in $25 \%, 22.1 \%, 3.6 \%$, and $2.9 \%$ of the infants, respectively, and $6.6 \%$ needed surgical intervention. The rate of late anemia was $45.7 \%$, with $26.4 \%$ requiring packed cell transfusion.

Conclusions: Our study revealed that the rate of regular post-discharge follow-ups of preterm infants was low. Regarding growth impairments of preterm neonates, special attention to nutrition is recommended after discharge from the hospital. Given the low rate of exclusive breastfeeding of premature infants, comprehensive planning to raise the rate of feeding with breast milk is very important. Regarding the high rate of anemia and the need for packed cell transfusion, paying attention to good nutrition and iron supplementation is crucial after discharge.
\end{abstract}

Keywords: Premature Neonates, Follow-Up, Growth Indices, Neonatal Intensive Care Unit

\section{Background}

Preterm delivery (before 37 weeks of gestation) in cludes $5 \%-15 \%$ of childbirths worldwide (1-3). A significant percentage of infant and neonatal mortality and morbidity is related to premature babies. Problems related to prematurity consists of respiratory, nutritional, neurodevelopmental, and gastrointestinal complications involving these patients from the time of delivery and hospital admission to the post-discharge duration at home. There is a crucial need for special care of preterm infants in neona- tal intensive care units (NICUs), as well as in post-discharge periods (4-6). The best outcomes of these patients are obtained by continuing the follow-up of their medical problems at special follow-up clinics. Regarding the need for following up on multiple problems of premature infants by multidisciplinary and sophisticated teams, usual health centers affiliated to the Health Ministry are not suitable for these complicated follow-ups (7). The special consideration of additive care and follow-ups for preterm neonates compared to term babies is done at high-risk neonatal follow-up clinics (HRNFCs). 
The HRFNCs are multidisciplinary clinics for the detection and management of problems in different fields such as nutritional, growth/developmental, neurological, hearing, visual, and rehabilitation aspects in preterm babies from early post-discharge periods to months or years after the discharge $(8,9)$. On the other hand, obtaining information about long-term morbidity of high-risk neonates at follow-up clinics can be helpful for the appropriate changing of standard care of these patients to achieve a better prognosis. At HRNFCs, parental training for better care of their children can be an important factor for the decline of long-term consequences of preterm delivery $(7,10)$. The American Academy of Pediatrics (AAP) suggested that the discharge planning of premature babies needs to contain six essential components including the education of parents, assessments of unresolved medical problems, performance of primary care, planning for home care, mobilization and identification of support services, and surveillance and designation of follow-up clinics (11).

\section{Objectives}

Considering the importance of post-discharge care and follow-up of preterm babies, we planned a study to investigate long-term and post-discharge medical problems of preterm babies delivered at our maternity hospital and admitted to our NICU.

\section{Methods}

The present study is a prospective descriptive study focusing on preterm infants delivered from 2016 to 2017 at Mahdieh Maternity Hospital. The inclusion criteria included all premature neonates with a gestational age of less than 34 weeks and birth weight of less than $2000 \mathrm{~g}$ admitted to the NICU of the hospital because of prematurity and low birth weight from among those who were alive at the time of discharge to home. The exclusion criteria included dead neonates or those transferred to another hospital with no follow-up at the HRNFC. The Ethics Committee of Shahid Beheshti University of Medical Sciences approved the study and written consent was taken from the parents of patients.

Based on the policy of the care of preterm neonates in our hospital, all patients were referred to the HRNFC after discharge from the NICU to visit and follow-up their main problems. As mentioned, all preterm neonates that had at least one visit to our HRNFC were enrolled in the study. The timing of the first post-discharge visit was determined by the attending neonatologist or special nurse of the neonate. The first visit was done usually $2-3$ days post-discharge. The frequency of outpatient visiting was weekly for all patients with a discharge weight of lower than 2,000 g, two weekly for infants weighing 2,000-3,000 $\mathrm{g}$, and monthly for infants weighing higher than $3,000 \mathrm{~g}$. Due to the lack of access to patients, in spite of suggestions to parents for the regular referral of their neonates to the HRNFC, the timing of follow-up visits was different for all infants. Regarding the importance of the growth pattern of preterm infants, we measured body weight, head circumference, and height of the neonates at all visits and the pattern of growth was assessed based on the special curve for preterm babies.

The hearing screening was done by auditory brainstem response (ABR) at centers out of the hospital, but the visual examination was done at our clinic by an expert ophthalmologist at retinopathy of prematurity (ROP). For infants with pallor detected in the physical examination, cell blood count $(\mathrm{CBC})$ was checked. For neonates with hemoglobin and hematocrit levels of lower than $7 \mathrm{mg} / \mathrm{dL}$ and $21 \mathrm{mg} / \mathrm{dL}$, respectively, despite the intake of a full dose of iron supplement, readmission in the neonatal ward was planned for packed cell transfusion.

By interviewing the mothers, the problems related to nutrition, consisting of the selection of the milk consumed, methods, frequency, and volumes of feeding, and the lactation technique, were checked and instructions were presented to the mothers, if needed. For babies with feeding intolerance, instructions were presented for changing the formula.

The vaccination and repeated screening for hypothyroidism were checked based on the protocols of the Health Ministry. Other possible problems of infants such as surgical problems, infantile colic, allergic symptoms, and gastroesophageal reflux were evaluated and managed. For all infants with spasticity detected by the physician or physiotherapist, rehabilitation and physiotherapy were done at special clinics of our hospital. Ultrasound of the brain, pelvis, and kidneys was done for all patients in the radiologic ward of the hospital, except for infants with a prolonged hospital stay or a previous normal finding of recent sonography. Developmental assessment by a special scoring system was not done at our HRFNC and for suspected cases with gross developmental delay, referral to a pediatric neurologist was done. Demographic data and anthropometric indices of the patients and all information about the mentioned issues were recorded, as well.

The collected data were analyzed by SPSS version 23 software. For the analysis of quantitative variables, we used the mean and standard deviation and for qualitative variables and multivariate analysis, a regression test was used. $\mathrm{P}<0.05$ was considered significant. 


\section{Results}

Based on the demographic data collected from 140 neonates, 72 (51.4\%) neonates were male and 68 (48.6\%) were female. The mean gestational age of the neonates was 30 weeks with a standard deviation of 2.36 . The lowest gestational age was 24 weeks. Table 1 shows the number of patients at different gestational ages. The mean birth weight of the neonates was 1,366.99 $\mathrm{g}$ with a standard deviation of 37.37. The lowest weight was $630 \mathrm{~g}$ and the highest weight was $1,990 \mathrm{~g}$. Table 1 shows the number of patients in different birth weight groups. The average length of stay of the patients in the NICU was 34.6 (5 - 140) days. The mean interval between the discharge and the referral time to the clinic was 6.16 days with a range of 1 to 45 days. The frequency of visits at the clinic is shown in Table 1 . The number of visits to the HRNFC at post-neonatal ages (after one month of life) is shown in Table 1. As can be seen, the number of visits to the HRNFC decreased with the increase in the age of patients and just 33 (23.5\%) patients referred to the clinic at the ages of three and six months.

\begin{tabular}{|c|c|}
\hline Variables & Values \\
\hline \multicolumn{2}{|c|}{ Gestational age, weeks of gestation } \\
\hline$<28$ & $35(25)$ \\
\hline $28-32$ & $81(57.9)$ \\
\hline$>32$ & $24(17.1)$ \\
\hline \multicolumn{2}{|l|}{ Birth weight, $g$} \\
\hline$<1000$ & $23(16.4)$ \\
\hline $1000-1500$ & $61(43.6)$ \\
\hline $1500-2000$ & $56(40)$ \\
\hline \multicolumn{2}{|l|}{ Number of visits to HRNFC } \\
\hline One time, the lowest & $14(10)$ \\
\hline 18 times, the highest & $2(1.4)$ \\
\hline$\geq 8$ times & $35(25)$ \\
\hline \multicolumn{2}{|c|}{ Number of visits to HRNFC at post-neonatal ages, mo } \\
\hline 3 & $60(42.8)$ \\
\hline 6 & $55(39.2)$ \\
\hline 12 & $14(10)$ \\
\hline \multicolumn{2}{|l|}{ Nutrition } \\
\hline Exclusive breast milk & $24(17.1)$ \\
\hline Formula and breast milk & $75(53.6)$ \\
\hline Exclusive formula & $41(29.3)$ \\
\hline
\end{tabular}

The nutrition selection of the patients is shown in Table 1 . Unfortunately, the number of exclusively breastfed infants was lower than the number of infants fed with formula. Among formula-fed infants, 97(69.3\%) cases were fed with preterm formula in the first few weeks after discharge and 5 (6.3\%) cases were fed with Post-Discharge Formula (PDF). In the absence of the PDF availability, after gaining the bodyweight of $3-3.5 \mathrm{~kg}$, the regular formula for term babies was recommended.

The results of the one-year follow-up of infants indicated that the rate of readmission was $26.4 \%(n=37)$. Infantile colic, gastroesophageal reflux, and respiratory allergy were reported in 35 (25\%), 31 (22.1\%), and 5 (3.6\%) cases, respectively, and 9 (6.4\%) cases needed surgical intervention for inguinal hernia. Prolonged Jaundice requiring readmission for phototherapy was detected in 10 (1.7\%) infants and cholestasis was observed in one case.

All newborns received the full vaccination as per the standard protocols and had a screening for hypothyroidism by filter papers at the age of $3-5,14,42$, and 70 days of life, based on the protocols of screening by the Health Ministry. In 4 (2.9\%) patients, hypothyroidism was detected and levothyroxine was administered for treatment.

Brain sonography was done in all 140 (100\%) eligible neonates before and after discharge and 122 (86.3\%) neonates had normal findings. Hydrocephaly and periventricular leukomalacia (PVL) were diagnosed in 11 (7.9\%) and 4 (2.9\%) neonates.

Echocardiography as a modality for the assessment of cardiac disorders was done in neonates with abnormal findings in the physical examination favoring cardiac dysfunction or in patients with previous abnormal echocardiography. In our patients, 3 (2.1\%) neonates had Patent Ductus Arteriosus (PDA) with no patients needing surgical closure of PDA. In 5 (3.6\%) neonates, other cardiac anomalies were recorded such as ASD and tetralogy of Fallot.

All preterm neonates admitted to the NICU were examined by ophthalmologists for retinopathy of prematurity (ROP) at the age of 4 weeks and repeated ophthalmologic examination was planned based on the maturity of retinal vascularity and suggestion of our ophthalmologist. After discharge from the hospital, all preterm infants had repeated exam for the diagnosis of ROP. Vascularization of the retina in 47 (33.6\%) infants were completely normal while the others had some degrees of the immaturity of retinal vascularity which needed repeated examination and 10 (17.1\%) infants had severe abnormality needing treatments such as intra-retinal injection of Avastin or laser therapy for prevention of blindness.

Based on the policy of our ward, all the neonates admitted to the neonatal ward and NICU should have hearing screening by otoacoustic emission (OAE), but because of the inaccessibility of ABR, as the main tool for the assessment of hearing loss in preterm infants, the hearing assess- 
ment was planned after discharge from the hospital. Unfortunately, just 42 (30\%) patients underwent this accurate evaluation, showing 39(92.8\%) normal findings and 3 (7.1\%) abnormal hearing findings.

For the screening of hip dislocation, 85 (60.8\%) neonates underwent a hip ultrasound at the ages of 4 - 6 weeks of life or later. Of them, 74 (87\%) cases had normal hip and 11 (12.9\%) cases had abnormal alpha (lower than 60 degrees) and beta angles related to type 1 A hip dislocation. In the follow-up of patients with probable hip dislocation, after a suggestion to parents for appropriate positioning of their neonates' hips, repeated hip sonography showed that all patients had normal hips.

Based on the reported discharge sheets of patients, 64 (45.7\%) neonates underwent pre-discharge hemoglobin evaluation and 37 (26.4\%) cases had mild anemia (hemoglobin $<10 \mathrm{mg} / \mathrm{dL}$ ) without the need for packed cell transfusion (before discharge) but after discharge, despite nutritional supplementation with iron, 66 (47.1\%) neonates had still anemia, 28 (20\%) of whom had severe anemia (hemoglobin $<7 \mathrm{mg} / \mathrm{dL}$ ) in need of readmission for packed cell transfusion.

All growth indices of the patients (birth weight, head circumference, and height) at birth are listed in Table 2. Based on the birth weight of our patients, infantile growth indices in the first, third, and sixth months of life are presented in Table 3.

\begin{tabular}{lccc}
\hline Table 2. Neonate Growth Indices at Birth & \\
\hline Percentile, \% & $\begin{array}{c}\text { Weight } \\
\text { Number, \% }\end{array}$ & $\begin{array}{c}\text { Height } \\
\text { Number, \% }\end{array}$ & $\begin{array}{c}\text { Head Circumference } \\
\text { Number, \% }\end{array}$ \\
\hline $\mathbf{3}$ & $21(15)$ & $30(21.6)$ & $16(11.55)$ \\
$\mathbf{1 0}$ & $43(30.7)$ & $34(24)$ & $42(30.2)$ \\
$\mathbf{5 0}$ & $59(42.1)$ & $50(36)$ & $52(37.4)$ \\
$\mathbf{9 0}$ & $16(11.4)$ & $21(15)$ & $26(18.7)$ \\
$\mathbf{9 7}$ & $1(0.7)$ & $3(2.2)$ & $2(1.4)$ \\
\hline
\end{tabular}

${ }^{\mathrm{a}}$ Values are expressed as No. (\%).

\section{Discussion}

Despite increasing the survival of premature neonates over the past few decades, the complications of prematurity still account for the main problems of the health system (12). Long-term morbidity of preterm infants requires multidisciplinary follow-ups for the prevention of delayed complications of prematurity or following the present involvements of them for better outcomes of these highrisk infants $(8,9)$. The establishment of special multidisciplinary clinics for preterm babies needs a team consist- ing of neonatologists, ophthalmologists, pediatric neurologists, physical therapists, audiologists, and nutritionists for following the main problems of them in each area for the prevention or treatment of long-term morbidities (7, 13,14).

The purpose of this study was to evaluate the postdischarge follow-up of preterm babies admitted to our NICU. A total of 140 premature infants were followed up for two years after discharge. According to the findings of this study, $25 \%$ of the newborns had more than eight visits, which shows desirable conditions. Unfortunately, 10\% had just one visit to our clinic, which can be an alarm for the healthcare system to assess the causes of parents' reluctance to bring their babies to clinics with the probability of missing their problems. Regarding the absence of a regionalization system for care at Iranian maternity hospitals, the place of residence of some patients in our NICU was far from our hospital and regular outpatient visiting of infants was not possible for parents.

The longest time interval of follow-up was 36 months observed just in one case. As mentioned in Table 1, 60 (42.8\%) patients had visited at their three months of life, 55 (39.2\%) at six months of life, and 14 (10\%) at one-year-old; therefore, it seems that the number of visits to our clinic decreased with increasing the age of patients. It is possible that due to the reduction of their specific problems over time, the continuing of their outpatient care was done at other general centers near to their residence locations.

Based on the growth indices at birth in our population, the most values of birth weight, height, and head circumference were at the 50th percentile of growth charts; that is, $42.1 \%$ of neonate birth weights, $36 \%$ of neonate heights, and $37.4 \%$ of neonate head circumferences were at the 50th percentile of growth charts. This issue indicates that due to good prenatal care of mothers, the number of premature infants appropriate for gestational age (AGA) was higher than growth retardation. In a study in Taiwan that was done in 2007 - 2011, Tsai et al. reported that AGA preterm infant delivery at birth was more than SGA (65\% vs. $33.2 \%$ ) (15).

Although the number of our neonates with AGA at birth was remarkable, the rate of growth impairments was significant after discharge from the hospital, particularly in extremely low birth weight infants (ELBW) with birth weight of less than $1000 \mathrm{~g}$ ( $\mathrm{n}=23$ infants) so that at the age of one month, $50 \%$ of the infants were at the 50 th percentile of weight while at the age of three months, $53 \%$ of the infants' weight and $40 \%$ of the infants' height were at the third percentile. Thus, this result indicates some degrees of growth impairment in these high-risk patients at a few months post-discharge. The justifying reasons for this issue can be the nutritional-related problems of these pa- 


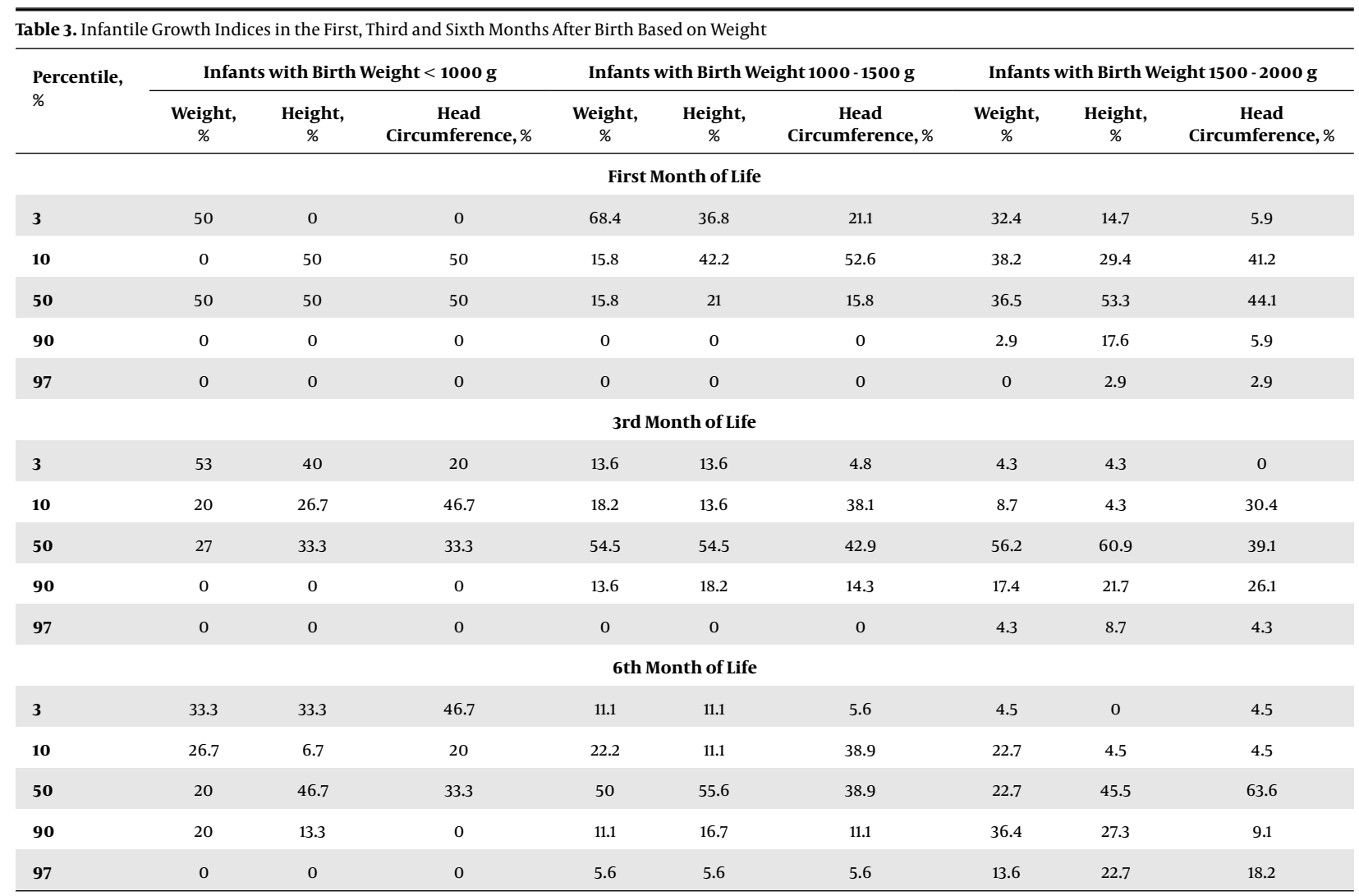

tients, such as the selection of milk, lack of proper lactation technique, use of unfortified breast milk, feeding with regular formula instead of a specific post-discharge formula for preterm infants, and gastrointestinal problems. However, at the age of six months, the number of patients at the third weight percentile decreased and $20 \%$ of the patients were at the 9oth percentile of weight.

Based on our results, a better condition was observed in neonates with a birth weight of 1,000 to $1,500 \mathrm{~g}$. Moreover, $54.5 \%$ by the age of three months and $50 \%$ at the age of six months were at the 50th weight percentile of the growth curve. In infants with a birth weight of more than $1,500 \mathrm{~g}$, the rate of increasing birth weight was more appropriate than in the neonates with lower-birth weight. Thus, ELBW infants needed more attention to the nutritional state. Our results are consistent with the results of a study by Modi et al. that showed weight, height, and head circumferences of newborns at 12 months of age increased compared to their birth time, but they had lower mean scores than term neonates (16).

The findings of our study indicated that $17.1 \%$ of the infants at the time of discharge were exclusively breastfeeding; 53.6\% were fed with breast milk and formula simultaneously and $29.3 \%$ were fed exclusively by the formula.
Despite the emphasis on the exclusive breastfeeding for all infants, especially preterm babies, nearly $83 \%$ of our infants did not feed exclusively by their breasts of mothers. Similar to our result, in a study by Rodrigues et al., $91 \%$ of newborns were non-exclusively breastfed (17). In a study by Lee and Jang (18) in 2016, exclusive breastfeeding in premature newborns from the first week to 12th week of life and breastfeeding concurrent with formula increased from $5.7 \%$ to $19.8 \%$ and from $27.3 \%$ to $67.9 \%$, respectively. Breastfeeding with formula at the same time decreased from $67 \%$ to $12.3 \%$. However, the increase in nutrition with formula was much higher than the increase in breastfeeding (18).

Given that 11 (7.9\%) hydrocephalus, 4 (2.9\%) PVL, and 3 (2.1\%) GMH patients were reported in our study, it seems that the investigation of neurological and developmental prematurity-induced complications is highly important and has a significant role in the healing of these patients. In Tsai et al. study in Taiwan, the rate of PVL was $0.33 \%$ at GA 30 - 32 weeks, $1.19 \%$ at GA 27 - 29 weeks, and 0.77 at GA 24 - 26 weeks (15).

One of the most important problems of prematurity is anemia. Prolonged hospitalization, lots of blood sampling, and the low levels of erythropoietin and iron stor- 
age of preterm infants are the leading causes of this problem before and after discharge from the hospital. The lack of attention to anemia can adversely affect the weight gain of infants or their neurological developments (19). In the present study, $47.1 \%$ of the neonates had post-discharge anemia and $20 \%$ of them required blood transfusions. Based on these results, it seems that the prevalence of postdischarge anemia is significantly high in premature infants. Therefore, paying more attention to the proper nutrition of premature infants and the use of food supplements, such as appropriate doses of iron drops, may prevent severe anemia and its consequences. These results are in line with the results of Domellof and Sjostrom's study in 2015 , in which $40 \%$ of preterm infants needed oral iron supplements after discharge from the hospital (20).

In the present study, 37 (26.4\%) infants had a history of re-admission to the hospital because of intensified jaundice (requiring phototherapy), respiratory distress, and the need for hernia repair surgery. Considering that all neonates in this study were premature with low birth weight, it seems that this number is acceptable.

According to Underwood et al. (21) study, approximately $15 \%$ of premature infants needed at least one hospitalization during their first year of life, with the highest prevalence being in neonates with the gestational age of fewer than 25 weeks. The most common cause of readmission was acute respiratory diseases (21).

One of the complications of prematurity is delayed hypothyroidism that can be detected in repeated screening tests. In the present study, delayed hypothyroidism requiring treatment with levothyroxine was detected in $2.9 \%$ of the neonates. In the study by Armanian et al., transient hypothyroidism was the most common endocrine problem in neonates with a prevalence of $33.33 \%$ (22).

One of the common problems of premature infants after discharge from the hospital is developmental delay and motor skill problems. In this study, $16.4 \%$ of newborns needed treatment with physical therapy. However, we did not use a standard assessment tool for the developmental evaluation of our patients, but based on routine physical examinations of the patients at the HRNFC or the reports of their parents, referring to a pediatric neurologist was suggested in cases with diagnosed or suspected developmental delay. Glass et al. (6) in 2015 reported high rates of neurodevelopmental abnormalities in preterm infants despite significant changes in the care of them. They also mentioned no improvement in the neurodevelopmental outcomes of preterm infants even though the incidence of some neurosensory impairments had been decreased (6).

A limitation of our study was the discordance of the time of visits of patients to the HRNFC. This irregularity in the interval of visiting our patients made the statistical analysis difficult and probably has caused inaccurate results of growth chart indices. Another limitation was the lack of planned neurodevelopmental assessments in our patients.

\subsection{Conclusions}

According to the results of this study, the follow-up of premature infants after discharge from the hospital is necessary by an expert multidisciplinary team. Growth and developmental problems, especially in neonates with birth weights of less than $1000 \mathrm{~g}$, seem to be more important than in those with higher birth weights and as a result, more nutritional care is needed in this specific birth weight group. Considering the low percentage of exclusive breastfeeding in premature infants, comprehensive planning to increase the rate of breastfeeding by mothers of preterm neonates is very crucial. Due to the high prevalence of anemia and the need for blood transfusion after discharge from the hospital, proper nutrition with milk and dietary supplements is necessary to prevent anemia and blood transfusion. Developmental assessment of discharged premature infants should be taken into account in the outpatient care program after discharge from the hospital.

\section{Acknowledgments}

The authors of this study would like to thank the staff of the NICU of Mahdieh Hospital.

\section{Footnotes}

Authors' Contribution: Study concept and design: Minoo Fallahi and Naeeme Taslimi. Acquisition of data: Minoo Fallahi, Naeeme Taslimi, and Zahra Soltanttooyeh Analysis and interpretation of data: Soltantooyeh, Shamshiri, and Minoo Fallahi. Drafting of the manuscript: Minoo Fallahi and Naeeme Taslimi. Critical revision of the manuscript for important intellectual content: Minoo Fallahi and Naeeme Taslimi. Statistical analysis: Ahmad Reza Shamshiri.

Conflict of Interests: The authors confirm that there is no relevant financial interest or financial conflict.

Ethical Approval: The study protocol was approved by the Ethics Committee of Shahid Beheshti University of Medical Sciences and written consent was obtained from the mothers.

Funding/Support: This project was not supported by any institute. 


\section{References}

1. Purisch SE, Gyamfi-Bannerman C. Epidemiology of preterm birth. Semin Perinatol. 2017;41(7):387-91. doi: 10.1053/j.semperi.2017.07.009. [PubMed: 28865982].

2. Vakilian K, Ranjbaran M, Khorsandi M, Sharafkhani N, Khodadost M. Prevalence of preterm labor in Iran: A systematic review and meta-analysis. Int J Reprod Biomed (Yazd). 2015;13(12):743-8. [PubMed: 27141533]. [PubMed Central: PMC4827509].

3. Torchin H, Ancel PY, Jarreau PH, Goffinet F. [Epidemiology of preterm birth: Prevalence, recent trends, short- and long-term outcomes]. J Gynecol Obstet Biol Reprod (Paris). 2015;44(8):723-31. doi: 10.1016/j.jgyn.2015.06.010. [PubMed: 26143095].

4. Afjeh SA, Sabzehei MK, Fallahi M, Esmaili F. Outcome of very low birth weight infants over 3 years report from an Iranian center. Iran J Pediatr. 2013;23(5):579-87. [PubMed: 24800021]. [PubMed Central: PMC4006510].

5. Mills J, Elgizoli B, Herath HMD. Problems of prematurity. InnovAiTEduc Inspiration Gen Pract. 2018;11(12):658-62. doi: 10.1177/1755738018782291.

6. Glass HC, Costarino AT, Stayer SA, Brett CM, Cladis F, Davis PJ. Outcomes for extremely premature infants. Anesth Analg. 2015;120(6):1337-51. doi: 10.1213/ANE.0000000000000705. [PubMed: 25988638]. [PubMed Central: PMC4438860].

7. Doyle LW, Anderson PJ, Battin M, Bowen JR, Brown N, Callanan C, et al. Long term follow up of high risk children: Who, why and how? BMC Pediatr. 2014;14:279. doi: 10.1186/1471-2431-14-279. [PubMed: 25399544]. [PubMed Central: PMC4289257].

8. Bockli K, Andrews B, Pellerite M, Meadow W. Trends and challenges in United States neonatal intensive care units follow-up clinics.J Perinatol. 2014;34(1):71-4. doi: 10.1038/jp.2013.136. [PubMed: 24177221].

9. Patra K, Greene MM, Perez B, Silvestri JM. Neonatal high-risk follow-up clinics: How to improve attendance in very low birth weight infants. EJ Neonatol Res. 2014;4(1):3-13.

10. Steinhardt A, Hinner P, Kuhn T, Roehr CC, Rudiger M, Reichert J. Influences of a dedicated parental training program on parent-child interaction in preterm infants. Early Hum Dev. 2015;91(3):205-10. doi: 10.1016/j.earlhumdev.2015.01.012. [PubMed: 25676187].

11. Kuo DZ, Lyle RE, Casey PH, Stille CJ. Care system redesign for preterm children after discharge from the NICU. Pediatrics. 2017;139(4). doi: 10.1542/peds.2016-2969. [PubMed: 28250024].
12. Lawn JE, Gravett MG, Nunes TM, Rubens CE, Stanton C; Gapps Review Group. Global report on preterm birth and stillbirth (1 of 7): Definitions, description of the burden and opportunities to improve data. BMC Pregnancy Childbirth. 2010;10 Suppl 1. S1. doi: 10.1186/1471-2393-10S1-S1. [PubMed: 20233382]. [PubMed Central: PMC2841772].

13. Ritchie SK. Primary care of the premature infant discharged from the neonatal intensive care unit. MCN Am J Matern Child Nurs. 2002;27(2):76-85. doi: 10.1097/00005721-200203000-00004. [PubMed: 11984275].

14. [No author listed]. Follow-up care of high-risk infants. Pediatrics. 2004;114(Supplement 5):1377-97. doi: 10.1542/peds.2004-0866.

15. Tsai LY, Chen YL, Tsou KI, Mu SC; Taiwan Premature Infant Developmental Collaborative Study Group. The impact of smallfor-gestational-age on neonatal outcome among very-lowbirth-weight infants. Pediatr Neonatol. 2015;56(2):101-7. doi: 10.1016/j.pedneo.2014.07.007. [PubMed: 25440777].

16. Modi M, Saluja S, Kler N, Batra A, Kaur A, Garg P, et al. Growth and neurodevelopmental outcome of VLBW infants at 1 year corrected age. Indian Pediatr. 2013;50(6):573-7. doi: 10.1007/s13312-013-0170-5. [PubMed: 23255692].

17. Rodrigues C, Teixeira R, Fonseca MJ, Zeitlin J, Barros H; Portuguese Epice Network. Prevalence and duration of breast milk feeding in very preterm infants: A 3-year follow-up study and a systematic literature review. Paediatr Perinat Epidemiol. 2018;32(3):237-46. doi: 10.1111/ppe.12457. [PubMed: 29469986].

18. Lee SY, Jang GJ. Prevalence and predictors of exclusive breastfeeding in late preterm infants at 12 weeks. Child Health Nurs Res. 2016;22(2):7986. doi: 10.4094/chnr.2016.22.2.79.

19. Grantham-McGregor S, Ani C. A review of studies on the effect of iron deficiency on cognitive development in children.J Nutr. 2001;131(2S2):649S-66S. discussion 666S-668S. doi: 10.1093/jn/131.2.649S. [PubMed: 11160596].

20. Domellof M, Sjostrom ES. Enteral iron supplementation in preterm infants. J Pediatr Gastroenterol Nutr. 2017;64(1). e26. doi: 10.1097/MPG.0000000000001445. [PubMed: 27782960].

21. Underwood MA, Danielsen B, Gilbert WM. Cost, causes and rates of rehospitalization of preterm infants. J Perinatol. 2007;27(10):614-9. doi: 10.1038/sj.jp.7211801. [PubMed: 17717521].

22. Armanian AM, Kelishadi R, Barekatain B, Salehimehr N, Feizi A. Frequency of thyroid function disorders among a population of verylow-birth-weight premature infants. Iran J Neonatol IJN. 2016;7(3):9-16. 